\title{
Increased Expression of Monocyte Chemoattractant Protein-1 in Atherectomy Specimens From Patients With Restenosis After Percutaneous Transluminal Coronary Angioplasty
}

\author{
Seiji Hokimoto, MD; Yuichi Oike, MD**; Taro Saito, MD; \\ Mitsuhiko Kitaoka, MD*; Shuichi Oshima, MD; Katsuo Noda, MD; \\ Yasushi Moriyama, MD; Fumiyuki Ishibashi, MD; Hisao Ogawa, MD ${ }^{\dagger}$
}

\begin{abstract}
The plasma concentration of monocyte chemoattractant protein-1 (MCP-1) antigen is higher in patients with restenosis after coronary angioplasty than in those who do not restenose. In this study the MCP-1 expression of coronary atherectomy specimens was investigated by immunohistochemistry. Samples were obtained from 12 patients with restenosis and 15 with de novo lesions by directional coronary atherectomy. MCP-1 immunoreactivity was found in all patients in the restenosis group and in 8 of the de novo group. The frequency of macrophage expression was higher in the restenosis group than in de novo group. These results indicate that local expression of MCP-1 may be associated with the mechanisms of vascular remodeling after coronary angioplasty. (Circ J 2002; 66: 114-116)
\end{abstract}

Key Words: Coronary artery disease; Immunohistochemistry; Restenosis

A though percutaneous transluminal coronary angioplasty (PTCA) is an established treatment for coronary artery disease (CAD) and its primary success rate is high, restenosis after the procedure is still a problem! Histological studies of the restenosed vessel reveal intimal hyperplasia, and similar histological findings have been observed in atherosclerotic lesions? Macrophages play an important role in the progression of atherosclerosis ${ }^{3}$ and in the clinical state of $\mathrm{CAD}_{4}^{4}$ and monocyte chemoattractant protein-1 (MCP-1) is found in the macrophage-rich areas of atherosclerosis 5 Recently, Furukawa et al reported that anti-MCP-1 antibody inhibits neointimal hyperplasia in injured rat carotid arteries and that MCP-1 is involved in the development of neointimal hyperplasia after vascular injury! In humans, we have already reported the relationship between the plasma concentration of MCP1 antigen and restenosis after balloon angioplasty? but the local, noncirculating MCP-1 expression remains unclear. Thus, in the present study we used immunohistochemistry to examine the MCP-1 expression in specimens obtained after directional coronary atherectomy (DCA) for restenosis after PTCA, and in de novo lesions.

The study group comprised 27 patients who had evidence of $90 \%$ or greater narrowing of a major coronary artery and the lesion anatomy made it suitable for DCA. The study group was divided into 2 groups: restenosis and de novo lesions. The restenosis after PTCA group consisted of 12

(Received September 18, 2001; revised manuscript received October 22, 2001; accepted November 13, 2001)

Divisions of Cardiology and *Pathology, Kumamoto Central Hospital, **Department of Cell Differentiation Institute of Molecular Embryology and Genetics, Kumamoto University and Department of Cardiovascular Medicine, Kumamoto University School of Medicine, Kumamoto, Japan

Mailing address: Seiji Hokimoto, MD, Division of Cardiology, Kumamoto Central Hospital, 1-5-1 Tainoshima, Kumamoto City, Kumamoto 862-0965, Japan. E-mail: seisan@ momo.so-net.ne.jp patients (10 men, 2 women, aged 48-66 years, mean 57 years) and the de novo group consisted of 15 patients (13 men, 2 women, aged $42-76$ years, mean age 58 years) who had typical exertional angina and had not received coronary intervention. The lesions identified for DCA were located in the left anterior descending coronary artery in 11 patients in the restenosis and in 13 in the de novo group, in the left circumflex coronary artery in 1 patient in each group and in the right coronary artery in 1 patient in the de novo group. Medication was given as usual: aspirin as the anti-platelet agent, nitrates and calcium antagonists.

The study protocol was in agreement with the guidelines of the ethical committee of the institution and each patient or their family gave written informed consent.

Hypertension was defined as systolic blood pressure $>160$ $\mathrm{mmHg}$ or diastolic blood pressure $>100 \mathrm{mmHg}$. Diabetic mellitus was defined according to WHO criteria. Hyperlipidemia was defined as serum total cholesterol $>220$ $\mathrm{mg} / \mathrm{dl}$ or LDL-cholesterol $>140 \mathrm{mg} / \mathrm{dl}$.

The following monoclonal antibodies were used for the immunohistochemistry: 5D3-F7 (anti-MCP-1, Pharmingen), KP-1 (anti-macrophage, Dako), and HHF35 (anti-smooth muscle actin, Dako).

The atherectomy specimens were embedded in OCT compound (Tissue-Tek, Miles Inc, Elkhart, IN, USA), quickly frozen in dry-ice acetone, and stored at $-80^{\circ} \mathrm{C}$ until use. The frozen sections were immunostained by the indirect immunoperoxidase method. After inhibition of endogenous peroxidase activity, the sections were stained with one of the antibodies listed and then reacted with peroxidaselabeled antimouse immunoglobulin. Peroxidase activity was visualized using 3,3'-diaminobenzidine as the substrate. After immunostaining, the slides were lightly counterstained with hematoxylin. For controls, the tissues were incubated with non-immunized mouse serum instead of specific antibodies and processed by the same procedure. To judge whether tissues were stained, each slide was eval- 
Table 1 Patient Characteristics

\begin{tabular}{lccc}
\hline \hline & $\begin{array}{c}\text { Restenosis } \\
(n=12)\end{array}$ & $\begin{array}{c}\text { de novo } \\
(n=15)\end{array}$ & $p$ value \\
\hline Age (years) & $57 \pm 3.0$ & $58 \pm 3.5$ & $N S$ \\
M/F & $10 / 2$ & $13 / 2$ & $N S$ \\
Hypertension & $4(33 \%)$ & $7(47 \%)$ & $N S$ \\
Diabetes & $5(42 \%)$ & $6(40 \%)$ & $N S$ \\
Smoking & $7(58 \%)$ & $9(60 \%)$ & $N S$ \\
Hyperlipidemia & $5(42 \%)$ & $6(40 \%)$ & $N S$ \\
Obesity & $2(17 \%)$ & $2(13 \%)$ & $N S$ \\
\hline
\end{tabular}

Values are mean \pm SEM. NS, not significant.

Table 2 Frequency of Positive Immunostaining for MCP-1, Smooth Muscle Cells and Macrophages

\begin{tabular}{lccc}
\hline \hline & $\begin{array}{c}\text { Restenosis } \\
(n=12)\end{array}$ & $\begin{array}{c}\text { de novo } \\
(n=15)\end{array}$ & $p$ value \\
\hline MCP-1 & $12 / 12(100 \%)$ & $8 / 15(53 \%)$ & $<0.05$ \\
Smooth muscle cell & $12 / 12(100 \%)$ & $15 / 15(100 \%)$ & $N S$ \\
Macrophage & $10 / 12(83 \%)$ & $8 / 15(53 \%)$ & $<0.05$ \\
\hline
\end{tabular}

NS, not significant.
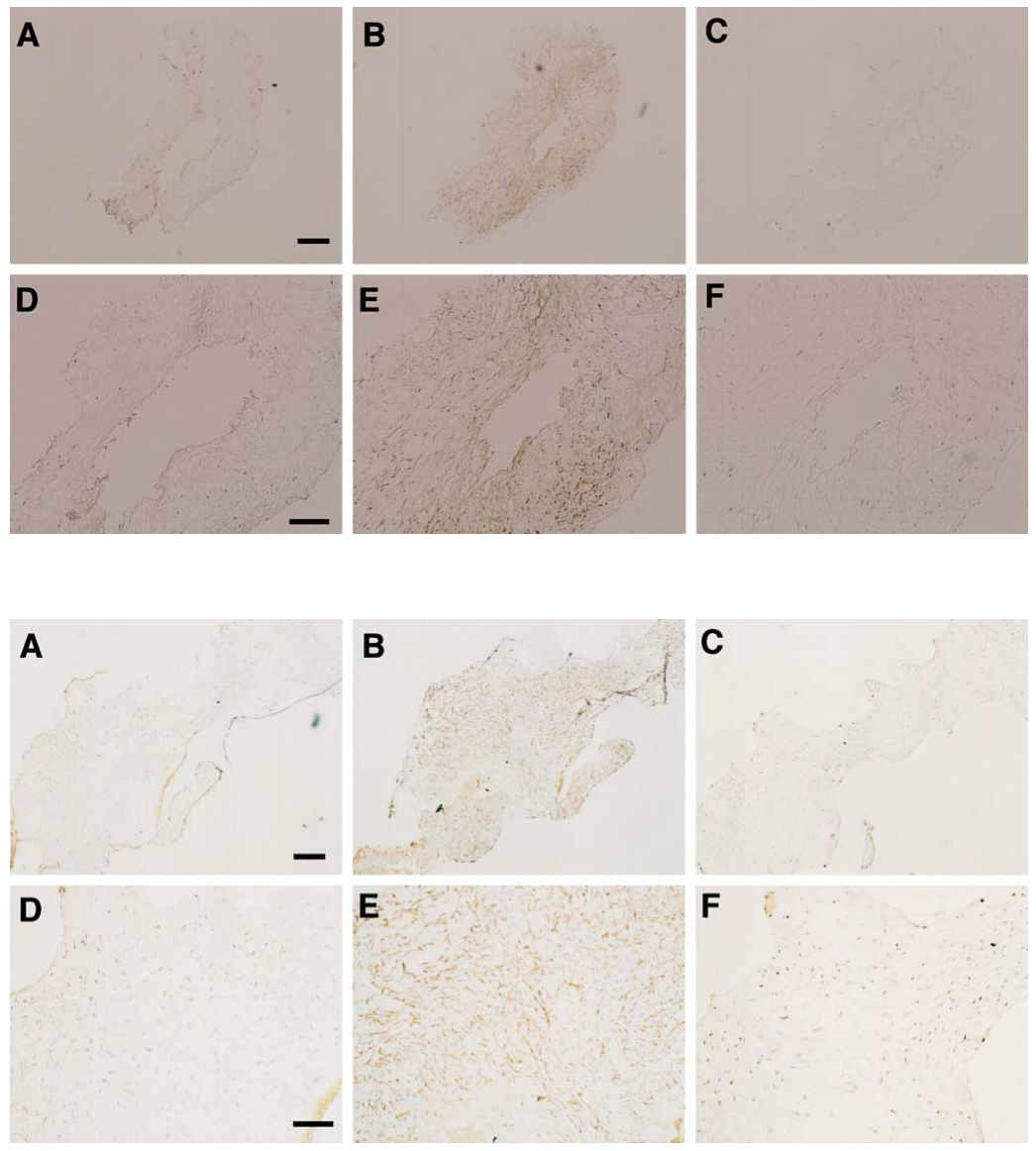

Fig 1. Immunohistochemical staining of serial sections of atherectomy specimen obtained from the de novo lesion of stable exertional anigina. (A) No immunoreactivity in the coronary plaque with anti-MCP-1 antibody. (B, C) Immunohistochemistry for smooth muscle cells and macrophages was performed with the same monoclonal antibody used in (A). Immunoreactivity for smooth muscle cells was observed (B), but not for macrophages (C). (D-F) Higher-magnification views of A-C, respectively. Bars $=200 \mu \mathrm{m}$ in $\mathrm{A}-\mathrm{C}$, and $100 \mu \mathrm{m}$ in D-F.

Fig 2. Immunohistochemical stainings of serial sections of atherectomy sample obtained from restenosis lesion after balloon angioplasty. Immunoreactivity for (A) MCP-1, (B, C) smooth muscle cells and macrophages. (D-F) Higher-magnification views of $A-C$, respectively. Bars $=200 \mu \mathrm{m}$ in $\mathrm{A}-\mathrm{C}$, and $100 \mathrm{\mu} \mathrm{m}$ in $\mathrm{D}-\mathrm{F}$. uated independently by 2 different observers, and the results given as positive or negative, when compared with those stained with non-immunized mouse serum.

There were no significant differences in the patients' characteristics of age, sex and coronary risk factors (Table 1). Table 2 shows the frequency of positive immunostaining for MCP-1, smooth muscle cell, and macrophage expression. In the de novo group, MCP-1 immunoreactivity was found in 8 of the 15 patients, and immunostaining for smooth muscle cell and macrophage were seen, respectively, in all subjects and 8 of 15 patients. Fig 1 is representative of the immunohistochemical staining of serial sections of atherectomy specimens obtained from a patient with the de novo lesion of stable exertional angina. Immunoreactivity for MCP-1 or macrophages was not observed, but there was smooth muscle cell immunostain- 
ing (Fig 1B,E). However, in the restenosis group, MCP-1 immunoreactivity was found in all lesions after PTCA (Fig 2A,D), immunostaining for smooth muscle cell was observed in all patients (Fig 2B,E) and immunoreactivity for macrophages was observed in 10 of the 12 subjects (Fig 2C,F). Moreover, immunostaining for MCP-1 was strongly observed around the macrophage infiltration.

MCP-1 is reported to be upregulated in human atherosclerotic plaque, in the arteries of primates on a hypercholesterolemic diet and in vascular endothelial and smooth muscle cells exposed to minimally modified lipids? Moreover, MCP-1 plays an important role in the development of early atherosclerotic lesions? Clinically, we have reported that plasma antigen levels of MCP-1 are higher in patients with acute coronary syndrome than in those with stable exertional angina ${ }^{10}$ and that patients with restenosis after PTCA have higher levels in the chronic phase compared with those without restenosis? These results indicate that the level of circulating MCP-1 reflects the clinical state, stable or not, of CAD and may be related to restenosis after balloon angioplasty. However, the histological findings of the human coronary artery are unclear so far.

In the present study, we used immunohistochemistry to examine the expression of MCP-1 in DCA specimens. Comparing restenosis lesions with de novo lesions, smooth muscle cells immunoreactivity was seen in all samples and the frequency of immunostaining for both MCP-1 and macrophage was higher in restenosis than in de novo lesions. Moreover, the results of immunostaining serial sections suggest that MCP-1 is expressed on some macrophages and smooth muscle cells, and in particular, strong immunostaining for MCP-1 was observed around the macrophage infiltration. Thus, the local MCP-1 level in the human coronary artery may be related to the vascular remodeling that occurs after balloon angioplasty, but it is unknown whether the relation between MCP-1 accumulation and restenosis after angioplasty is primary or secondary. Moreover, it remains unknown which types of smooth muscle cells express MCP-1. Further studies are needed.

\section{Acknowledgments}

This study was supported in part by a Grant-in-Aid for Scientific Research C11670692 from the Ministry of Education, Science, Sports and Culture, Tokyo, and Research Grant for Cardiovascular Disease (10C-5, 11C-6) from the Ministry of Health and Welfare.

We thank cytotechnologists, Yoshiteru Oka, Toshihiro Tateyama, Madoka Takeda and Toshifumi Nishimura for their help. We are grateful to Namiko Sakamoto and Eiko Kikuchi for their assistance with collecting the data.

\section{References}

1. Holmes DR Jr, Vlietstra RE, Smith HC, Vetrovec GW, Kent KM, Cowley MJ, et al. Restenosis after percutaneous transluminal coronary angioplasty (PTCA): A report from the PTCA registry of the national heart, lung, and blood institute. Am J Cardiol 1984; 53: 77C-81C.

2. Liu M, Roubin G, King S. Restenosis after coronary angioplasty: Potential biologic determinants and role of intimal hyperplasia. Circulation 1989; 79: 1374-1387.

3. Ross R. The pathogenesis of atherosclerosis: A perspective for the 1990s. Nature 1993; 362: 801-809.

4. Kaikita K, Ogawa H, Yasue H, Takeya M, Takahashi K, Saito T, et al. Tissue factor expression on macrophages in coronary plaques in patients with unstable angina. Arterioscler Thromb Vasc Biol 1997; 17: $2232-2237$.

5. Nelken N, Coughlin S, Gordon D, Wilcox J. Monocyte chemoattractant protein-1 in human atheromatous plaques. J Clin Invest 1991; 88: $1121-1127$

6. Furukawa Y, Matsumori A, Ohashi N, Shioi T, Harada A, Matsushima $\mathrm{K}$, et al. Anti-monocyte chemoattractant protein-1/monocyte chemotactic and activating factor antibody inhibits neointimal hyperplasia in injured rat carotid arteries. Circ Res 1999; 84: 306-314.

7. Hokimoto S, Ogawa H, Saito T, Oshima S, Noda K, Soejima H, et al. Increased plasma antigen levels of monocyte chemoattractant protein1 in patients with restenosis after percutaneous transluminal coronary angioplasty. Jpn Circ J 2000; 64: 831-834.

8. Cushing SD, Berliner JA, Valente AJ, Territo MC, Navab M, Parhami F, et al. Minimally modified low density lipoprotein induces monocyte chemotactic protein 1 in human endothelial cells and smooth muscle cell. Proc Natl Acad Sci USA 1990; 87: 5134-5138.

9. Boring L, Gosling J, Cleary M, Charo I. Decreased lesion formation in CCR2-1- mice reveals a role for chemokines in the initiation of atherosclerosis. Nature 1998; 394: 894-897.

10. Nishiyama K, Ogawa H, Yasue H, Soejima H, Misumi K, Takazoe $\mathrm{K}$, et al. Simultaneous elevation of the levels of circulating monocyte chemoattractant protein-1 and tissue factor in acute coronary syndromes. Jpn Circ J 1998; 62: 710-712. 